\title{
Effective collision strengths for transitions in Ni XIX ${ }^{\star}$
}

\author{
K. M. Aggarwal and F. P. Keenan
}

\author{
Astrophysics Research Centre, School of Mathematics and Physics, Queen's University Belfast, Belfast BT7 1NN, \\ Northern Ireland, UK \\ e-mail: K.Aggarwal@qub.ac.uk
}

Received 13 May 2008 / Accepted 13 June 2008

\section{ABSTRACT}

\begin{abstract}
Aims. In this paper we report electron impact collision strengths and excitation rates for transitions among the lowest 89 levels of Ni XIX.

Methods. The Dirac atomic $R$-matrix code (DARC) is adopted for the calculations of collision strengths and subsequently the effective collision strengths.

Results. Collision strengths for resonance transitions among 89 levels arising from the $\left(1 s^{2}\right) 2 s^{2} 2 p^{6}, 2 s^{2} 2 p^{5} 3 \ell, 2 s 2 p^{6} 3 \ell, 2 s^{2} 2 p^{5} 4 \ell$, and $2 \mathrm{~s} 2 \mathrm{p}^{6} 4 \ell$ configurations of Ni XIX are reported over a wide energy range below 250 Ryd. Additionally, effective collision strengths for all 3916 transitions among the 89 levels are listed over a wide temperature range below $10^{7} \mathrm{~K}$. Comparisons are made among different calculations and the accuracy of the data is assessed. Finally, comparisons between theoretical and experimental intensity ratios of some prominent lines of Ni XIX are discussed.
\end{abstract}

Key words. atomic data - atomic processes

\section{Introduction}

Iron group elements ( $\mathrm{Sc}$ to $\mathrm{Zn}$ ) are highly useful for the modelling and diagnosing of a variety of plasmas, such as astrophysical, fusion and laser-generated. However, highly accurate atomic data including for energy levels, radiative rates, collision strengths, and excitation rates are normally required for such work. Among iron group elements, Fe is the most abundant and important, and we have already reported calculations for many of its ions (IX-XXVI: see Aggarwal et al. 2008, and references therein). After Fe, nickel is the most abundant element and many emission lines of its ions, including those from Ni XIX, have been observed in the Sun - see, for example, Jupen (1984) and Feldman et al. (2000). Similarly many transitions, particularly within the $n=3$ configurations, have been measured in laboratory plasmas by Feldman et al. (1967), Swartz et al. (1971), Boiko et al. (1977), Buchet et al. (1987), Biémont et al. (2000), and more recently by $\mathrm{Gu}$ et al. (2004, 2007). Therefore, to fulfill the requirement of atomic data, we have also reported calculations for Ni XI-XVII (Aggarwal et al. 2003, 2007; and Aggarwal $\&$ Keenan 2007c, 2008), and in this paper we focus our attention on Ne-like Ni XIX.

Ne-like ions, particularly Fe XVII and Ni XIX, show a rich spectrum from solar and other astrophysical plasmas. For example, emission lines of Ni XIX have been observed by the Chandra X-ray satellite in Galactic black holes (Miller et al. 2005) and active galactic nuclei (Krongold et al. 2003). Additionally, the determination of $\mathrm{Ni}$ abundance is important in studies of supernova explosions and gamma ray bursts (Lucy 1995; Reeves et al. 2002). Similarly, many lines of nickel ions, including Ni XIX, have been measured in laboratory plasmas,

* Table 3 is only available in electronic form at the CDS via anonymous ftp to cdsarc.u-strasbg. fr $(130.79 .128 .5)$ or via http://cdsweb.u-strasbg.fr/cgi-bin/qcat? J/A+A/488/365 particularly in the 9-15 A wavelength range (Brown et al. 2001; $\mathrm{Gu}$ et al. 2004, 2007). Of particular recent interest are the six resonance lines of $\mathrm{Ni}$ XIX, namely $3 \mathrm{C}\left(2 \mathrm{p}^{6}{ }^{1} \mathrm{~S}_{0}-2 \mathrm{p}^{5} 3 \mathrm{~d}{ }^{1} \mathrm{P}_{1}^{\circ}: 1-\right.$ 27), 3D (2 $\left.\mathrm{p}^{6}{ }^{1} \mathrm{~S}_{0}-2 \mathrm{p}^{5} 3 \mathrm{~d}^{3} \mathrm{D}_{1}^{\circ}: 1-23\right), 3 \mathrm{E}\left(2 \mathrm{p}^{6}{ }^{1} \mathrm{~S}_{0}-2 \mathrm{p}^{5} 3 \mathrm{~d}^{3} \mathrm{P}_{1}^{\circ}\right.$ : $1-17), 3 \mathrm{~F}\left(2 \mathrm{p}^{6}{ }^{1} \mathrm{~S}_{0}-2 \mathrm{p}^{5} 3 \mathrm{~s}{ }^{3} \mathrm{P}_{1}^{\circ}\right.$ : $\left.1-5\right), 3 \mathrm{G}\left(2 \mathrm{p}^{6}{ }^{1} \mathrm{~S}_{0}-2 \mathrm{p}^{5} 3 \mathrm{~s}{ }^{1} \mathrm{P}_{1}^{\circ}\right.$ : $1-3)$, and $3 \mathrm{H}\left(2 \mathrm{p}^{6}{ }^{1} \mathrm{~S}_{0}-2 \mathrm{p}^{5} 3 \mathrm{~s}^{3} \mathrm{P}_{2}^{\circ}: 1-2\right)$, at respective wavelengths of $12.434 \AA$, $12.658 \AA, 12.810 \AA, 13.779 \AA, 14.043 \AA$, and $14.077 \AA$ - see Table 1 for level indices. The last transition $3 \mathrm{H}$ is also referred to as M2 as listed by $\mathrm{Gu}$ et al. (2007). These prominent strong lines have been observed in the $\mathrm{X}$ ray spectra of solar and stellar coronae, active galactic nuclei, $\mathrm{X}$-ray binaries, and supernovae from the Chandra and XMMNewton satellites. Furthermore, line intensity ratios have also been measured on EBIT (electron beam ion trap) machines at the Lawrence Livermore National Laboratory (LLNL) by Brown et al. (2001) and $\mathrm{Gu}$ et al. (2004, 2007), and at the National Institute of Standards and Technology (NIST) by Chen et al. (2006b). To provide theoretical support to experimental work, a few calculations have also appeared recently, such as by $\mathrm{Gu}$ et al. (2004) and Chen et al. (2006a,b). However, these calculations are limited to comparisons between the theoretical and experimental line intensity ratios, and do not report any atomic data.

Considering the importance of Ni XIX, there have been a few calculations in the past, particularly for the determination of energy levels and radiative rates ( $A$-values). Aggarwal \& Keenan (2006b) have recently reviewed the available data among 89 levels of the $\left(1 \mathrm{~s}^{2}\right) 2 \mathrm{~s}^{2} 2 \mathrm{p}^{6}, 2 \mathrm{~s}^{2} 2 \mathrm{p}^{5} 3 \ell, 2 \mathrm{~s} 2 \mathrm{p}^{6} 3 \ell, 2 \mathrm{~s}^{2} 2 \mathrm{p}^{5} 4 \ell$, and $2 \mathrm{~s}^{2} \mathrm{p}^{6} 4 \ell$ configurations, and have reported $A$-values for four types of transitions, namely electric dipole (E1), magnetic dipole (M1), electric quadrupole (E2), and magnetic quadrupole (M2). These are the most comprehensive sets of radiative rates available to date for transitions in $\mathrm{Ni}$ XIX. However, for the other important atomic parameter, namely 
Table 1. Target levels of Ni XIX and their threshold energies (in Ryd).

\begin{tabular}{|c|c|c|c|c|c|c|c|}
\hline Index & "Configuration & $\overline{\text { Level }}$ & $\overline{\text { GRASP }}$ & Index & Configuration & Level & $\overline{\overline{\text { GRASP }}}$ \\
\hline 1 & $2 s^{2} 2 p^{6}$ & ${ }^{1} \mathrm{~S}_{0}$ & 0.00000 & 46 & $2 s^{2} 2 p^{5} 4 s$ & ${ }^{3} \mathrm{P}_{1}^{\circ}$ & 88.51422 \\
\hline 2 & $2 s^{2} 2 p^{5} 3 s$ & ${ }^{3} \mathrm{P}_{2}^{\circ}$ & 64.59266 & 47 & $2 s^{2} 2 p^{5} 4 p$ & ${ }^{3} \mathrm{P}_{0}$ & 88.81253 \\
\hline 3 & $2 s^{2} 2 p^{5} 3 s$ & ${ }^{1} \mathrm{P}_{1}^{\circ}$ & 64.75556 & 48 & $2 s^{2} 2 p^{5} 4 p$ & ${ }^{3} \mathrm{D}_{1}$ & 89.58303 \\
\hline 4 & $2 s^{2} 2 p^{5} 3 s$ & ${ }^{3} \mathrm{P}_{0}^{\circ}$ & 65.89549 & 49 & $2 s^{2} 2 p^{5} 4 d$ & ${ }^{3} \mathrm{P}_{0}^{\circ}$ & 89.62341 \\
\hline 5 & $2 s^{2} 2 p^{5} 3 s$ & ${ }^{3} \mathrm{P}_{1}^{\circ}$ & 65.99248 & 50 & $2 s^{2} 2 p^{5} 4 d$ & ${ }^{3} \mathrm{P}_{1}^{\circ}$ & 89.66194 \\
\hline 6 & $2 s^{2} 2 p^{5} 3 p$ & ${ }^{3} \mathrm{~S}_{1}$ & 67.11863 & 51 & $2 s^{2} 2 p^{5} 4 d$ & ${ }^{3} \mathrm{~F}_{4}^{\circ}$ & 89.71042 \\
\hline 7 & $2 s^{2} 2 p^{5} 3 p$ & ${ }^{3} \mathrm{D}_{2}$ & 67.38277 & 52 & $2 s^{2} 2 p^{5} 4 d$ & ${ }^{3} \mathrm{P}_{2}^{\circ}$ & 89.72552 \\
\hline 8 & $2 s^{2} 2 p^{5} 3 p$ & ${ }^{3} \mathrm{D}_{3}$ & 67.57916 & 53 & $2 s^{2} 2 p^{5} 4 d$ & ${ }^{3} \mathrm{~F}_{3}^{\circ}$ & 89.72977 \\
\hline 9 & $2 s^{2} 2 p^{5} 3 p$ & ${ }^{1} \mathrm{P}_{1}$ & 67.65968 & 54 & $2 s^{2} 2 p^{5} 4 p$ & ${ }^{3} \mathrm{P}_{1}$ & 89.70737 \\
\hline 10 & $2 s^{2} 2 p^{5} 3 p$ & ${ }^{3} \mathrm{P}_{2}$ & 67.82370 & 55 & $2 s^{2} 2 p^{5} 4 p$ & ${ }^{1} \mathrm{D}_{2}$ & 89.72619 \\
\hline 11 & $2 s^{2} 2 p^{5} 3 p$ & ${ }^{3} \mathrm{P}_{0}$ & 68.36453 & 56 & $2 s^{2} 2 p^{5} 4 d$ & ${ }^{1} \mathrm{D}_{2}^{\circ}$ & 89.78376 \\
\hline 12 & $2 s^{2} 2 p^{5} 3 p$ & ${ }^{3} \mathrm{D}_{1}$ & 68.63561 & 57 & $2 s^{2} 2 p^{5} 4 d$ & ${ }^{3} \mathrm{D}_{3}^{\circ}$ & 89.81961 \\
\hline 13 & $2 s^{2} 2 p^{5} 3 p$ & ${ }^{3} \mathrm{P}_{1}$ & 68.95945 & 58 & $2 s^{2} 2 p^{5} 4 p$ & ${ }^{1} \mathrm{~S}_{0}$ & 89.96517 \\
\hline 14 & $2 s^{2} 2 p^{5} 3 p$ & ${ }^{1} \mathrm{D}_{2}$ & 69.00116 & 59 & $2 s^{2} 2 p^{5} 4 d$ & ${ }^{3} \mathrm{D}_{1}^{\circ}$ & 90.05354 \\
\hline 15 & $2 s^{2} 2 p^{5} 3 p$ & ${ }^{1} \mathrm{~S}_{0}$ & 70.13098 & 60 & $2 s^{2} 2 p^{5} 4 f$ & ${ }^{3} \mathrm{D}_{1}$ & 90.37202 \\
\hline 16 & $2 s^{2} 2 p^{5} 3 d$ & ${ }^{3} \mathrm{P}_{0}^{\circ}$ & 70.91200 & 61 & $2 s^{2} 2 p^{5} 4 f$ & ${ }^{1} \mathrm{G}_{4}$ & 90.37912 \\
\hline 17 & $2 s^{2} 2 p^{5} 3 d$ & ${ }^{3} \mathrm{P}_{1}^{\circ}$ & 71.00144 & 62 & $2 s^{2} 2 p^{5} 4 f$ & ${ }^{3} \mathrm{G}_{5}$ & 90.38262 \\
\hline 18 & $2 s^{2} 2 p^{5} 3 d$ & ${ }^{3} \mathrm{P}_{2}^{\circ}$ & 71.16951 & 63 & $2 s^{2} 2 p^{5} 4 f$ & ${ }^{3} \mathrm{D}_{2}$ & 90.38653 \\
\hline 19 & $2 s^{2} 2 p^{5} 3 d$ & ${ }^{3} \mathrm{~F}_{4}^{\circ}$ & 71.16811 & 64 & $2 s^{2} 2 p^{5} 4 f$ & ${ }^{3} \mathrm{~F}_{3}$ & 90.41809 \\
\hline 20 & $2 s^{2} 2 p^{5} 3 d$ & ${ }^{3} \mathrm{~F}_{3}^{\circ}$ & 71.22664 & 65 & $2 s^{2} 2 p^{5} 4 f$ & ${ }^{1} \mathrm{D}_{2}$ & 90.42322 \\
\hline 21 & $2 s^{2} 2 p^{5} 3 d$ & ${ }^{1} \mathrm{D}_{2}^{\circ}$ & 71.37793 & 66 & $2 s^{2} 2 p^{5} 4 f$ & ${ }^{1} \mathrm{~F}_{3}$ & 90.42724 \\
\hline 22 & $2 s^{2} 2 p^{5} 3 d$ & ${ }^{3} \mathrm{D}_{3}^{\circ}$ & 71.47633 & 67 & $2 s^{2} 2 p^{5} 4 f$ & ${ }^{3} \mathrm{~F}_{4}$ & 90.43562 \\
\hline 23 & $2 s^{2} 2 p^{5} 3 d$ & ${ }^{3} \mathrm{D}_{1}^{\circ}$ & 71.91932 & 68 & $2 s^{2} 2 p^{5} 4 d$ & ${ }^{3} \mathrm{~F}_{2}^{\circ}$ & 91.02549 \\
\hline 24 & $2 s^{2} 2 p^{5} 3 d$ & ${ }^{3} \mathrm{~F}_{2}^{\circ}$ & 72.51867 & 69 & $2 s^{2} 2 p^{5} 4 d$ & ${ }^{3} \mathrm{D}_{2}^{\circ}$ & 91.05187 \\
\hline 25 & $2 s^{2} 2 p^{5} 3 d$ & ${ }^{3} \mathrm{D}_{2}^{\circ}$ & 72.59210 & 70 & $2 s^{2} 2 p^{5} 4 d$ & ${ }^{1} \mathrm{~F}_{3}^{\circ}$ & 91.07845 \\
\hline 26 & $2 s^{2} 2 p^{5} 3 d$ & ${ }^{1} \mathrm{~F}_{3}^{\circ}$ & 72.64864 & 71 & $2 s^{2} 2 p^{5} 4 d$ & ${ }^{1} \mathrm{P}_{1}^{\circ}$ & 91.25686 \\
\hline 27 & $2 s^{2} 2 p^{5} 3 d$ & ${ }^{1} \mathrm{P}_{1}^{\circ}$ & 73.24505 & 72 & $2 s^{2} 2 p^{5} 4 f$ & ${ }^{3} \mathrm{G}_{3}$ & 91.70349 \\
\hline 28 & $2 \mathrm{~s} 2 \mathrm{p}^{6} 3 \mathrm{~s}$ & ${ }^{3} \mathrm{~S}_{1}$ & 75.91019 & 73 & $2 s^{2} 2 p^{5} 4 f$ & ${ }^{3} \mathrm{G}_{4}$ & 91.71542 \\
\hline 29 & $2 s^{2} p^{6} 3 s$ & ${ }^{1} \mathrm{~S}_{0}$ & 76.45810 & 74 & $2 s^{2} 2 p^{5} 4 f$ & ${ }^{3} \mathrm{~F}_{2}$ & 91.72231 \\
\hline 30 & $2 s 2 p^{6} 3 p$ & ${ }^{3} \mathrm{P}_{0}^{\circ}$ & 78.62091 & 75 & $2 s^{2} 2 p^{5} 4 f$ & ${ }^{3} \mathrm{D}_{3}$ & 91.72243 \\
\hline 31 & $2 s^{2} p^{6} 3 p$ & ${ }^{3} \mathrm{P}_{1}^{\circ}$ & 78.66211 & 76 & $2 s 2 p^{6} 4 s$ & ${ }^{3} S_{1}$ & 98.34835 \\
\hline 32 & $2 s 2 p^{6} 3 p$ & ${ }^{3} \mathrm{P}_{2}^{\circ}$ & 78.91529 & 77 & $2 s 2 p^{6} 4 s$ & ${ }^{1} \mathrm{~S}_{0}$ & 98.54064 \\
\hline 33 & $2 s^{2} p^{6} 3 p$ & ${ }^{1} \mathrm{P}_{1}^{\circ}$ & 79.06836 & 78 & $2 s 2 p^{6} 4 p$ & ${ }^{3} \mathrm{P}_{0}^{\circ}$ & 99.45014 \\
\hline 34 & $2 \mathrm{~s} 2 \mathrm{p}^{6} 3 \mathrm{~d}$ & ${ }^{3} \mathrm{D}_{1}$ & 82.35964 & 79 & $2 s 2 p^{6} 4 p$ & ${ }^{3} \mathrm{P}_{1}^{\circ}$ & 99.46456 \\
\hline 35 & $2 \mathrm{~s} 2 \mathrm{p}^{6} 3 \mathrm{~d}$ & ${ }^{3} \mathrm{D}_{2}$ & 82.37523 & 80 & $2 s^{2} p^{6} 4 p$ & ${ }^{3} \mathrm{P}_{2}^{\circ}$ & 99.56767 \\
\hline 36 & $2 \mathrm{~s} 2 \mathrm{p}^{6} 3 \mathrm{~d}$ & ${ }^{3} \mathrm{D}_{3}$ & 82.40539 & 81 & $2 s 2 p^{6} 4 p$ & ${ }^{1} \mathrm{P}_{1}^{\circ}$ & 99.61892 \\
\hline 37 & $2 s 2 p^{6} 3 d$ & ${ }^{1} \mathrm{D}_{2}$ & 82.82932 & 82 & $2 s^{2} p^{6} 4 d$ & ${ }^{3} \mathrm{D}_{1}$ & 100.86169 \\
\hline 38 & $2 s^{2} 2 p^{5} 4 s$ & ${ }^{3} \mathrm{P}_{2}^{\circ}$ & 87.18001 & 83 & $2 s 2 p^{6} 4 d$ & ${ }^{3} \mathrm{D}_{2}$ & 100.86875 \\
\hline 39 & $2 s^{2} 2 p^{5} 4 s$ & ${ }^{1} \mathrm{P}_{1}^{\circ}$ & 87.23366 & 84 & $2 s^{2} p^{6} 4 d$ & ${ }^{3} \mathrm{D}_{3}$ & 100.88248 \\
\hline 40 & $2 s^{2} 2 p^{5} 4 p$ & ${ }^{3} \mathrm{~S}_{1}$ & 88.23420 & 85 & $2 \mathrm{~s} 2 \mathrm{p}^{6} 4 \mathrm{~d}$ & ${ }^{1} \mathrm{D}_{2}$ & 101.03066 \\
\hline 41 & $2 s^{2} 2 p^{5} 4 p$ & ${ }^{3} \mathrm{D}_{2}$ & 88.29789 & 86 & $2 s 2 p^{6} 4 f$ & ${ }^{3} \mathrm{~F}_{2}^{\circ}$ & 101.51947 \\
\hline 42 & $2 s^{2} 2 p^{5} 4 p$ & ${ }^{3} \mathrm{D}_{3}$ & 88.37966 & 87 & $2 s 2 p^{6} 4 f$ & ${ }^{3} \mathrm{~F}_{3}^{\circ}$ & 101.52125 \\
\hline 43 & $2 s^{2} 2 p^{5} 4 p$ & ${ }^{1} \mathrm{P}_{1}$ & 88.40888 & 88 & $2 s 2 p^{6} 4 f$ & ${ }^{3} \mathrm{~F}_{4}^{\circ}$ & 101.52723 \\
\hline 44 & $2 s^{2} 2 p^{5} 4 p$ & ${ }^{3} \mathrm{P}_{2}$ & 88.46323 & 89 & $2 s 2 p^{6} 4 f$ & ${ }^{1} \mathrm{~F}_{3}^{\circ}$ & 101.54040 \\
\hline 45 & $2 s^{2} 2 p^{5} 4 s$ & ${ }^{3} \mathrm{P}_{0}^{\circ}$ & 88.48770 & & & & \\
\hline
\end{tabular}

collision strengths $(\Omega)$, the notable results available in the literature are by Zhang et al. (1987), Zhang \& Sampson (1989), and Mohan et al. (1993). Zhang et al. adopted the CoulombBorn-exchange (CBE) method and reported values of $\Omega$ for resonance transitions of many Ne-like ions, including Ni XIX. They employed the non-relativistic hydrogenic basis states in order to perform rapid calculations for many ions. Subsequently, Zhang \& Sampson improved the accuracy of their earlier work by employing more accurate relativistic atomic structure in the well-known and widely-used distorted-wave (DW) method. However, their calculations still remained confined to resonance transitions, whereas data for all transitions are required for the modelling of plasmas, as demonstrated by Del Zanna et al. (2004). Additionally, Zhang \& Sampson reported values of $\Omega$ at only a few energies above thresholds, whereas it is well known that closed-channel (Feshbach) resonances contribute significantly to the determination of effective collision strengths (or equivalently excitation rates), especially for the forbidden transitions. Therefore, their reported data for $\Omega$ are of limited applications.

Mohan et al. (1993) adopted the configuration interaction (CI) wavefunctions in an $R$-matrix approach 
(Berrington et al. 1978). They performed their calculations in $L S$ coupling and reported values of $\Omega$ in a limited energy range below 140 Ryd, but for all transitions among the lowest 15 states of the $2 s^{2} 2 p^{6}$ and $2 s^{2} 2 p^{5} 3 l$ configurations. Furthermore, they resolved resonances in thresholds region and reported values of effective collision strengths $(\Upsilon)$ over a wide electron temperature range up to $10^{7} \mathrm{~K}$. Since it is the fine-structure transitions which are observed spectroscopically, they extended their calculations to report values of $\Omega$ for 10 fine-structure transitions among the levels of the $2 s^{2} 2 p^{6}$ and $2 s^{2} 2 p^{5} 3$ s configurations (Singh \& Mohan 2005). Unfortunately, both their calculations in $L S$ and $L S J$ coupling, for values of $\Omega$ as well as $\Upsilon$, are inaccurate for many transitions, as discussed and demonstrated by Aggarwal \& Keenan (2006a, 2007a,b). Therefore, we will not discuss their results any further in this paper, and will only focus on our present calculations and those available earlier by Zhang \& Sampson (1989).

In the present work, we focus on the lowest 89 levels of the $2 \mathrm{~s}^{2} 2 \mathrm{p}^{6}, 2 \mathrm{~s}^{2} 2 \mathrm{p}^{5} 3 \ell, 2 \mathrm{~s} 2 \mathrm{p}^{6} 3 \ell, 2 \mathrm{~s}^{2} 2 \mathrm{p}^{5} 4 \ell$, and $2 \mathrm{~s} 2 \mathrm{p}^{6} 4 \ell$ configurations of Ni XIX, i.e. the same levels as considered by Zhang \& Sampson (1989), and for which we have already reported $A$-values (Aggarwal \& Keenan 2006b). Additionally, our approach is fully relativistic, as we employ the GRASP (generalpurpose relativistic atomic structure package) and Dirac atomic $R$-matrix code (DARC) for the calculations of wavefunctions and scattering process, respectively.

\section{Collision strengths}

The $2 s^{2} 2 p^{6}, 2 s^{2} 2 p^{5} 3 \ell, 2 s 2 p^{6} 3 \ell, 2 s^{2} 2 p^{5} 4 \ell$, and $2 s^{2} p^{6} 4 \ell$ configurations of $\mathrm{Ni}$ XIX give rise to 89 fine-structure levels, listed in Table 1. For our calculations, we have adopted the GRASP code, which was originally developed by Grant et al. (1980) but has recently been updated by Dr. Norrington. Additionally, we have used the option of extended average level (EAL), in which a weighted (proportional to $2 j+1$ ) trace of the Hamiltonian matrix is minimized. This produces a compromise set of orbitals describing closely lying states with moderate accuracy. The calculations also include corrections from the Breit and QED effects. A detailed comparison of our calculated energy levels with the experimental compilations of NIST (http://physics.nist. gov/PhysRefData) and available theoretical results has already been made in our earlier paper (Aggarwal \& Keenan 2006b), and hence will not be repeated here. Energy levels listed in Table 1 were assessed to be accurate to better than $1 \%$, and the reported $A$-values accurate to better than $20 \%$ for a majority of the strong transitions with oscillator strengths ( $f$-values) $\geq 0.01$. Finally, the inclusion of additional CI with the $n=5$ configurations made a negligible effect on the energy levels as well as the $A$ values, and therefore our calculations for collision strengths include only the above listed 89 levels.

For the calculations of collision strengths, we have employed the DARC program of Norrington \& Grant (private communication), which includes the relativistic effects in a systematic way, in both the target description and the scattering model. It is based on the $j j$ coupling scheme, and uses the Dirac-Coulomb Hamiltonian in the $R$-matrix approach. However, because of the inclusion of fine-structure in the definition of channel coupling, the matrix size of the Hamiltonian increases substantially. The $R$-matrix radius has been adopted to be 3.64 au, and 25 continuum orbitals have been included for each channel angular momentum for the expansion of the wavefunction. This allows us to compute $\Omega$ up to an energy of 250 Ryd. The maximum number of channels for a partial wave is 401 , and the corresponding size of the Hamiltonian matrix is 10086 . In order to obtain convergence of $\Omega$ for all transitions and at all energies, we have included all partial waves with angular momentum $J \leq 39.5$, although a higher range would have been preferable, particularly for the convergence of allowed transitions. However, to account for higher neglected partial waves, we have included a topup, based on the Coulomb-Bethe approximation (Burgess et al. 1970) for allowed transitions and geometric series for forbidden transitions.

In Figs. $1-3$ we show the variation of $\Omega$ with angular momentum $J$ at three energies of 150, 200, and 250 Ryd, and for three transitions, namely $1-27\left(2 \mathrm{~s}^{2} 2 \mathrm{p}^{6}{ }^{1} \mathrm{~S}_{0}-2 \mathrm{~s}^{2} 2 \mathrm{p}^{5} 3 \mathrm{~d}{ }^{1} \mathrm{P}_{1}^{\circ}\right)$, 2-6 $\left(2 s^{2} 2 p^{5} 3 s^{3} \mathrm{P}_{2}^{\circ}-2 s^{2} 2 p^{5} 3 p^{3} S_{1}\right)$, and $3-21\left(2 s^{2} 2 p^{5} 3 s^{1} P_{1}^{\circ}-\right.$ $2 \mathrm{~s}^{2} 2 \mathrm{p}^{5} 3 \mathrm{~d}{ }^{1} \mathrm{D}_{2}^{\circ}$, which are resonance, allowed, and forbidden, respectively. For all resonance transitions, our adopted range of partial waves is sufficient for the convergence of $\Omega$ at all energies, as shown in Fig. 1. However, for many allowed and some forbidden transitions among excited levels, our range of $J \leq 39.5$ is insufficient for the convergence of $\Omega$, as shown in Figs. 2 and 3. For such transitions a top-up has been included as stated above. Furthermore, for some transitions, such as the $2-6\left(2 s^{2} 2 p^{5} 3 s^{3} P_{2}^{\circ}-2 s^{2} 2 p^{5} 3 p^{3} S_{1}\right)$ shown in Fig. 2, the partial $\Omega$ show an unphysical oscillation for some random partial waves. This is due to the fact that the long-range potentials have been omitted for these partial waves and only Coulomb functions have been used. This is done automatically by the program in stage DSTGF whenever there is numerical problem in the convergence of the wavefunction in the outer region of the $R$-matrix. The solution to this problem is to increase the number of integration points. In the present calculations, we have kept this number to be 2000 , and increasing it further makes the program consuming more computational time. This is not currently feasible keeping in view our resources and the fact that $\Omega$ have to be computed at a large number of energies. However, as a result of this $\Omega_{J}$ is up to $20 \%$ lower than what it should be with the inclusion of long-range potentials. Since it happens for only two partial waves, the net effect on $\sum \Omega_{J}$ is negligible. For example, for the transition shown in Fig. 2, $\Omega$ are affected (and are lower) by less than $1 \%$, which is much less than the accuracy estimates of the calculations.

In Table 2 we present our results of $\Omega$ for all resonance transitions over a wide energy range (110 $\leq E \leq 250 \mathrm{Ryd})$, but above thresholds. The indices adopted to represent a transition are given in Table 1 . These results for $\Omega$ are not directly applicable in any modelling work, but are very useful in assessing the accuracy of a calculation. Earlier similar results for resonance transitions are available from the DW calculations of Zhang \& Sampson (1989), as stated in Sect. 1. For these transitions there are no discrepancies between our calculations and those of Zhang \& Sampson. Since no other similar results for transitions among excited levels are available, we have performed another calculation from the Flexible Atomic Code (FAC) of $\mathrm{Gu}$ (2003), which is available from the website http:// kipac-tree.standford.edu/fac. This is also a fully relativistic code like DARC, and is based on the DW method. Values of $\Omega$ from FAC are also included in Table 2 at a single excited $\left(E_{j}\right)$ energy of $\sim 145$ Ryd, which nearly corresponds to the highest (incident) energy of our calculations from DARC. Furthermore, similar values of $\Omega$ at three more energies of 59, 281, and 813 Ryd have already been listed in our earlier paper (Aggarwal \& Keenan 2007a).

In Fig. 4a we compare our values of $\Omega$ from the DARC and FAC codes for two important resonance transitions, namely 3D: $1-23\left(2 \mathrm{~s}^{2} 2 \mathrm{p}^{6}{ }^{1} \mathrm{~S}_{0}-2 \mathrm{~s}^{2} 2 \mathrm{p}^{5} 3 \mathrm{~d}{ }^{3} \mathrm{D}_{1}^{\circ}\right)$ and 


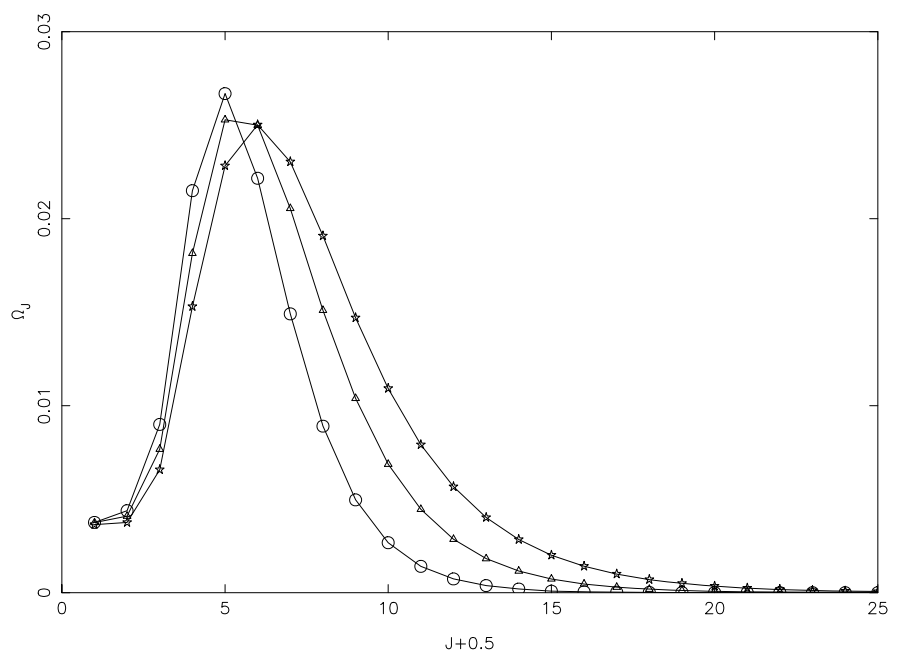

Fig. 1. Partial collision strengths for the $2 s^{2} 2 p^{6}{ }^{1} S_{0}-2 s^{2} 2 p^{5} 3 d{ }^{1} P_{1}^{\circ}$ (1-27) transition of Ni XIX, at three energies of: 150 Ryd (circles), 200 Ryd (triangles), and 250 Ryd (stars).

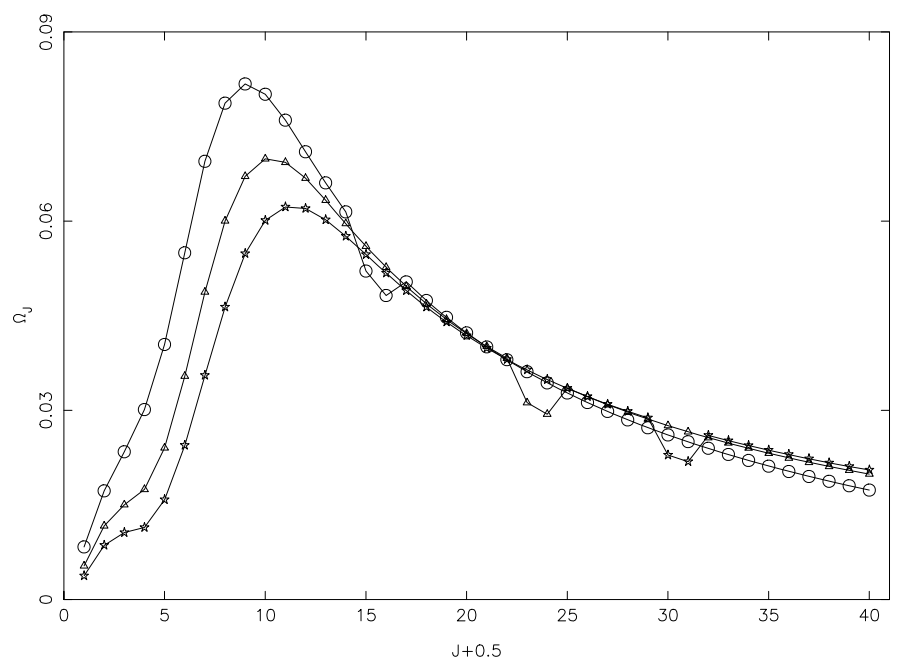

Fig. 2. Partial collision strengths for the $2 s^{2} 2 p^{5} 3 s{ }^{3} P_{2}^{\circ}-2 s^{2} 2 p^{5} 3 p{ }^{3} S_{1}$ (2-6) transition of Ni XIX, at three energies of: 150 Ryd (circles), 200 Ryd (triangles), and 250 Ryd (stars).

3C: $1-27\left(2 s^{2} 2 p^{6}{ }^{1} S_{0}-2 s^{2} 2 p^{5} 3 d{ }^{1} P_{1}^{\circ}\right)$. Also shown in this figure are the earlier results of Zhang \& Sampson (1989) and Chen et al. (2006a). For both of these (and many other) transitions, there is a complete agreement between our calculations from DARC and FAC and those of Zhang \& Sampson. However, for the 1-27 transition the earlier results of Chen et al. are underestimated by $\sim 10 \%$, over the entire common energy range. This is an important transition as already discussed and affects the ratio of the $3 \mathrm{C}$ and $3 \mathrm{D}$ lines. Considering that Chen et al. have also used the same DARC code as adopted by us, and have included a comparable range of partial waves $(J \leq 30.5$ which is fully sufficient for the convergence of $\Omega$ values for the 1-27 transition as shown in Fig. 1), a difference of $10 \%$ between the two calculations, for an important allowed transition, is not expected. The only major difference between our calculations and those of Chen et al. is that they have included levels of the $n=5$ configurations whereas we have not. However, this should not affect the calculations of $\Omega$, because the $f$-values obtained from the $n=4$ and $n=5$ calculations for the 1-27 transition agree within $1 \%$ - see Table 5 of Aggarwal \& Keenan (2006b) and Fig. 4 of

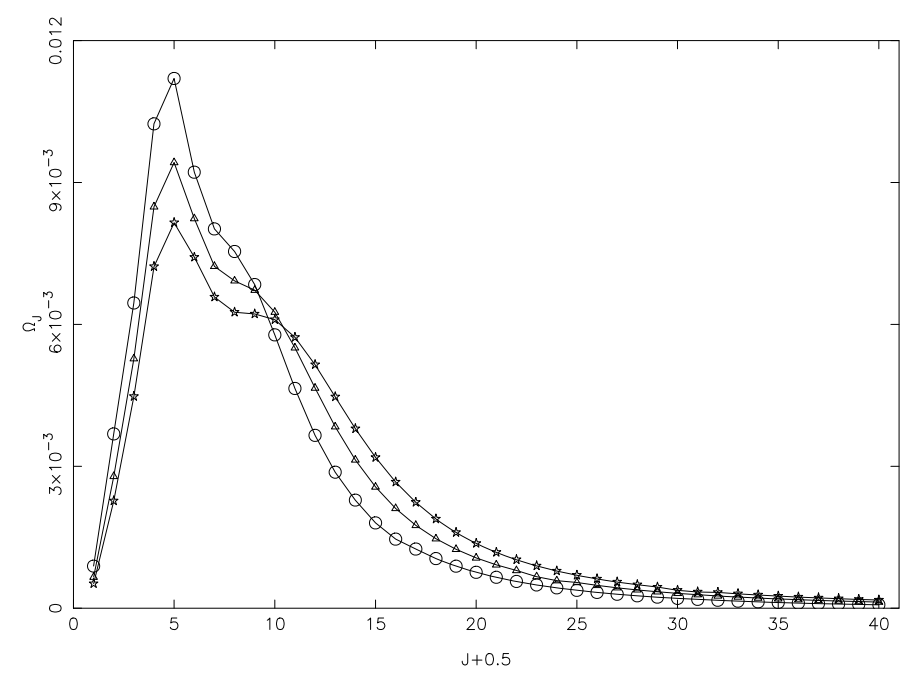

Fig. 3. Partial collision strengths for the $2 s^{2} 2 p^{5} 3 s^{1} P_{1}^{\circ}-2 s^{2} 2 p^{5} 3 d{ }^{1} D_{2}^{\circ}$ (3-21) transition of Ni XIX, at three energies of: 150 Ryd (circles), 200 Ryd (triangles), and 250 Ryd (stars).

Gu et al. (2004). A more extensive CI included in our calculations from FAC and the earlier work of Hibbert et al. (1993) does lower the $f$-value by $\sim 7 \%$, but there is no evidence that Chen et al. have included more CI than that included in the (GRASP2: $n \leq 5$ ) calculations of Aggarwal \& Keenan. In the absence of details about the calculations of Chen et al. it is difficult to pinpoint the source of difference between the two sets of $\Omega$ values.

In Fig. 4b we make a similar comparison of our values of $\Omega$ from the DARC and FAC codes for two forbidden transitions, namely $1-2\left(2 \mathrm{~s}^{2} 2 \mathrm{p}^{6}{ }^{1} \mathrm{~S}_{0}-2 \mathrm{~s}^{2} 2 \mathrm{p}^{5} 3 \mathrm{~s}^{3} \mathrm{P}_{2}^{\circ}\right)$ and $1-18\left(2 \mathrm{~s}^{2} 2 \mathrm{p}^{6}{ }^{1} \mathrm{~S}_{0}-\right.$ $\left.2 s^{2} 2 p^{5} 3 d^{3} \mathrm{P}_{2}^{\circ}\right)$. For the $1-2(3 \mathrm{H})$ transition, there is no discrepancy between our calculations and those of Chen et al. (2006a). Similarly values of $\Omega$ from our DW calculations from FAC and the earlier work of Zhang \& Sampson (1989) agree with DARC, particularly at higher energies. Differences at lower energies are $\sim 10 \%$. In general, we may state with confidence that all calculations, from both $R$-matrix and DW methods, agree within $\sim 10 \%$ for all resonance transitions, and this is also our assessment of accuracy for the values of $\Omega$ listed in Table 2 .

In Fig. 5a we compare our values of $\Omega$ from the DARC and FAC codes for three allowed transitions among excited levels, namely $2-13\left(2 s^{2} 2 p^{5} 3 s^{3} \mathrm{P}_{2}^{\circ}-2 s^{2} 2 p^{5} 3 p^{3} \mathrm{P}_{1}\right), 5-11\left(2 s^{2} 2 p^{5} 3 s\right.$ $\left.{ }^{3} \mathrm{P}_{1}^{\circ}-2 \mathrm{~s}^{2} 2 \mathrm{p}^{5} 3 \mathrm{p}^{3} \mathrm{P}_{0}\right)$, and $8-18\left(2 \mathrm{~s}^{2} 2 \mathrm{p}^{5} 3 \mathrm{p}^{3} \mathrm{D}_{3}-2 \mathrm{~s}^{2} 2 \mathrm{p}^{5} 3 \mathrm{~d}^{3} \mathrm{P}_{2}^{\circ}\right)$. For these three (and many other allowed) transitions, the agreement between the two calculations is better than $10 \%$. A similar comparison is shown in Fig. $5 \mathrm{~b}$ for three forbidden transitions, namely $2-16\left(2 \mathrm{~s}^{2} 2 \mathrm{p}^{5} 3 \mathrm{~s}^{3} \mathrm{P}_{2}^{\circ}-2 \mathrm{~s}^{2} 2 \mathrm{p}^{5} 3 \mathrm{~d}^{3} \mathrm{P}_{0}^{\circ}\right), 6-7\left(2 \mathrm{~s}^{2} 2 \mathrm{p}^{5} 3 \mathrm{p}^{3} \mathrm{~S}_{1}-\right.$ $\left.2 s^{2} 2 p^{5} 3 p{ }^{3} D_{2}\right)$, and $10-11\left(2 s^{2} 2 p^{5} 3 p{ }^{3} P_{2}-2 s^{2} 2 p^{5} 3 p{ }^{3} P_{0}\right)$. For these three (and many other) transitions also, there is no major discrepancy between our calculations from DARC and FAC. However, the agreement between the two calculations generally improves with increasing energy, as can be noted for the 6-7 and 10-11 transitions.

Since we have adopted a wide range of partial waves in order to obtain the convergence of $\Omega$ for the forbidden as well as the allowed transitions, have included a top-up to account for the contribution of higher neglected partial waves, and do not observe any (major) discrepancy with the DW calculations from FAC, the accuracy of our results for $\Omega$ for resonance transitions, listed in Table 2, is judged to be better than $10 \%$ as discussed 
Table 2. Collision strengths for resonance transitions of Ni XIX. $\left(a \pm b \equiv a \times 10^{ \pm b}\right)$.

\begin{tabular}{|c|c|c|c|c|c|c|c|}
\hline \multicolumn{2}{|c|}{ Transition } & \multicolumn{6}{|c|}{ Energy (Ryd) } \\
\hline$i$ & $j$ & 110 & 130 & 150 & 200 & 250 & $\mathrm{FAC}$ \\
\hline 1 & 2 & $1.062-03$ & $8.629-04$ & $7.154-04$ & $4.666-04$ & $3.273-04$ & $3.802-04$ \\
\hline 1 & 3 & $2.983-03$ & $3.608-03$ & $4.240-03$ & $5.619-03$ & $6.850-03$ & $5.743-03$ \\
\hline 1 & 4 & $2.128-04$ & $1.733-04$ & $1.436-04$ & $9.387-05$ & $6.587-05$ & $7.653-05$ \\
\hline 1 & 5 & $2.288-03$ & $2.714-03$ & $3.153-03$ & $4.128-03$ & $5.011-03$ & $4.380-03$ \\
\hline 1 & 6 & $2.332-03$ & $1.939-03$ & $1.596-03$ & $1.078-03$ & $7.743-04$ & $9.659-04$ \\
\hline 1 & 7 & $2.891-03$ & $2.839-03$ & $2.808-03$ & $2.842-03$ & $2.922-03$ & $3.398-03$ \\
\hline 1 & 8 & $2.510-03$ & $2.035-03$ & $1.642-03$ & $1.057-03$ & $7.314-04$ & $8.814-04$ \\
\hline 1 & 9 & $1.007-03$ & $8.192-04$ & $6.598-04$ & $4.264-04$ & $2.958-04$ & $3.157-04$ \\
\hline 1 & 10 & $2.449-03$ & $2.465-03$ & $2.490-03$ & $2.613-03$ & $2.748-03$ & $3.310-03$ \\
\hline 1 & 11 & $3.642-03$ & $3.735-03$ & $3.777-03$ & $3.885-03$ & $3.941-03$ & $3.473-03$ \\
\hline 1 & 12 & $1.034-03$ & $8.412-04$ & $6.770-04$ & $4.376-04$ & $3.033-04$ & $3.445-04$ \\
\hline 1 & 13 & $1.095-03$ & 8.937-04 & $7.192-04$ & $4.657-04$ & $3.228-04$ & $3.818-04$ \\
\hline 1 & 14 & $2.842-03$ & $2.836-03$ & $2.840-03$ & $2.940-03$ & $3.064-03$ & $3.715-03$ \\
\hline 1 & 15 & $3.546-02$ & $3.697-02$ & $3.766-02$ & $3.942-02$ & $4.033-02$ & $4.001-02$ \\
\hline 1 & 16 & $1.132-03$ & 8.814-04 & $7.006-04$ & $4.287-04$ & $2.849-04$ & $3.408-04$ \\
\hline 1 & 17 & $3.571-03$ & $2.942-03$ & $2.502-03$ & $1.881-03$ & $1.592-03$ & $1.613-03$ \\
\hline 1 & 18 & $4.149-03$ & $3.210-03$ & $2.536-03$ & $1.534-03$ & $1.010-03$ & $1.221-03$ \\
\hline 1 & 19 & $3.750-03$ & $2.855-03$ & $2.216-03$ & $1.295-03$ & $8.331-04$ & $1.003-03$ \\
\hline 1 & 20 & $3.230-03$ & -03 & $2.695-03$ & $2.454-03$ & $2.382-03$ & $3.237-03$ \\
\hline 1 & 21 & $1.501-03$ & $1.120-03$ & $8.548-04$ & $4.836-04$ & $3.031-04$ & $3.310-04$ \\
\hline 1 & 22 & 2.38 & 2.2 & $2.224-03$ & $2.246-03$ & -03 & -03 \\
\hline 1 & 23 & $3.458-02$ & -02 & $4.363-02$ & $5.306-02$ & 6.09 & -02 \\
\hline 1 & 24 & 1.8 & & $1.053-03$ & $6.093-04$ & & -04 \\
\hline 1 & 25 & 2.4 & & $1.452-03$ & $8.539-04$ & 5.50 & -04 \\
\hline 1 & 26 & & & $2.614-03$ & $2.552-03$ & -03 & -03 \\
\hline 1 & 27 & 9.5 & 1.09 & $1.218-01$ & $1.492-01$ & 1.7 & -01 \\
\hline 1 & 28 & 5.99 & & $3.783-04$ & $2.419-04$ & 1.6 & -04 \\
\hline 1 & 29 & & & $1.519-02$ & $1.613-02$ & 1.6 & -02 \\
\hline 1 & 30 & 1.79 & & $1.187-04$ & $7.782-05$ & 5.4 & -05 \\
\hline 1 & 31 & 1.0 & & $1.174-03$ & $1.452-03$ & 1.7 & -03 \\
\hline 1 & 32 & 8.8 & 7.2 & $6-04$ & 3.878 & 2.7 & -04 \\
\hline 1 & 33 & & & -03 & 7.408 & 9.4 & -03 \\
\hline 1 & 34 & & & -04 & 4.605 & 04 & -04 \\
\hline 1 & 35 & 1.9 & & $1.293-03$ & 8.41 & $6 .($ & -04 \\
\hline 1 & 36 & & & -03 & 1.06 & 04 & -04 \\
\hline 1 & 37 & & & -02 & 2.38 & 02 & -02 \\
\hline 1 & 38 & 4.8 & 3.7 & $3.003-04$ & 1.87 & -04 & -04 \\
\hline 1 & 39 & & & -04 & 6.97 & 04 & -04 \\
\hline 1 & 40 & & & 04 & 3.26 & & -04 \\
\hline 1 & 41 & 8.6 & 04 & -04 & 6.0 & 04 & -04 \\
\hline 1 & 42 & 1.1 & 04 & $2-04$ & 4.40 & -04 & -04 \\
\hline 1 & 43 & 4.7 & & 04 & 1.82 & 04 & -04 \\
\hline 1 & 44 & 6.8 & 6. & 04 & 5.0 & 04 & -04 \\
\hline 1 & 45 & 9.9 & -05 & $3-05$ & 3.7 & 05 & -05 \\
\hline 1 & 46 & 4.0 & -04 & & 5.1 & 04 & -04 \\
\hline 1 & 47 & 4.6 & 4.7 & 4.8 & 5.1 & 03 & -03 \\
\hline 1 & 48 & 5.02 & 3.9 & $3.103-04$ & 1.89 & 1.2 & -04 \\
\hline 1 & 49 & $4.891-04$ & $3.760-04$ & $2.946-04$ & $1.770-04$ & 1.16 & $1.115-04$ \\
\hline 1 & 50 & $1.406-03$ & $1.125-03$ & $9.267-04$ & $6.542-04$ & $5.250-04$ & 4.881-04 \\
\hline 1 & 51 & $1.817-03$ & $1.359-03$ & $1.032-03$ & $5.858-04$ & $3.691-04$ & $3.448-04$ \\
\hline 1 & 52 & $1.618-03$ & $1.224-03$ & $9.475-04$ & $5.551-04$ & $3.580-04$ & $3.418-04$ \\
\hline 1 & 53 & $1.142-03$ & $9.279-04$ & $7.885-04$ & $6.257-04$ & $5.664-04$ & $5.341-04$ \\
\hline 1 & 54 & $5.468-04$ & $4.303-04$ & $3.409-04$ & $2.100-04$ & $1.427-04$ & $1.410-04$ \\
\hline 1 & 55 & $8.367-04$ & $7.375-04$ & $6.685-04$ & $5.893-04$ & $5.658-04$ & $5.440-04$ \\
\hline 1 & 56 & $7.848-04$ & $5.712-04$ & $4.263-04$ & $2.325-04$ & $1.426-04$ & $1.157-04$ \\
\hline 1 & 57 & $8.005-04$ & $6.442-04$ & $5.528-04$ & $4.604-04$ & 4.409-04 & $4.087-04$ \\
\hline 1 & 58 & $6.699-03$ & $6.968-03$ & $7.176-03$ & $7.494-03$ & $7.789-03$ & $7.574-03$ \\
\hline 1 & 59 & $1.527-02$ & $1.761-02$ & $1.960-02$ & $2.402-02$ & $2.758-02$ & $2.562-02$ \\
\hline 1 & 60 & $2.069-04$ & $1.408-04$ & $1.008-04$ & $5.091-05$ & $2.978-05$ & $3.313-05$ \\
\hline 1 & 61 & $2.918-04$ & $2.447-04$ & $2.213-04$ & $2.038-04$ & $2.055-04$ & $2.007-04$ \\
\hline 1 & 62 & $3.519-04$ & $2.334-04$ & $1.640-04$ & $8.122-05$ & $4.777-05$ & $5.157-05$ \\
\hline
\end{tabular}


Table 2. Collision strengths for transitions in Ni XIX. $\left(a \pm b \equiv a \times 10^{ \pm b}\right)$.

\begin{tabular}{crllllll}
\hline \hline \multicolumn{2}{c}{ Transition } & & \multicolumn{5}{c}{ Energy (Ryd) } \\
$i$ & $j$ & 110 & 130 & 150 & 200 & 250 & FAC \\
\hline 1 & 63 & $5.915-04$ & $5.684-04$ & $5.747-04$ & $6.332-04$ & $7.008-04$ & $6.415-04$ \\
1 & 64 & $2.797-04$ & $1.866-04$ & $1.310-04$ & $6.370-05$ & $3.626-05$ & $4.073-05$ \\
1 & 65 & $9.763-04$ & $1.149-03$ & $1.309-03$ & $1.656-03$ & $1.928-03$ & $1.824-03$ \\
1 & 66 & $1.733-04$ & $1.131-04$ & $7.790-05$ & $3.666-05$ & $2.059-05$ & $1.886-05$ \\
1 & 67 & $1.881-04$ & $1.359-04$ & $1.079-04$ & $7.985-05$ & $7.268-05$ & $7.338-05$ \\
1 & 68 & $9.467-04$ & $7.041-04$ & $5.333-04$ & $3.005-04$ & $1.884-04$ & $1.676-04$ \\
1 & 69 & $1.378-03$ & $1.036-03$ & $7.975-04$ & $4.616-04$ & $2.951-04$ & $2.795-04$ \\
1 & 70 & $1.024-03$ & $8.342-04$ & $7.140-04$ & $5.796-04$ & $5.363-04$ & $4.981-04$ \\
1 & 71 & $1.497-02$ & $1.730-02$ & $1.927-02$ & $2.370-02$ & $2.725-02$ & $2.394-02$ \\
1 & 72 & $1.985-04$ & $1.309-04$ & $9.128-05$ & $4.431-05$ & $2.561-05$ & $2.623-05$ \\
1 & 73 & $2.435-04$ & $1.900-04$ & $1.618-04$ & $1.351-04$ & $1.302-04$ & $1.290-04$ \\
1 & 74 & $6.743-04$ & $7.577-04$ & $8.413-04$ & $1.038-03$ & $1.199-03$ & $1.130-03$ \\
1 & 75 & $3.129-04$ & $2.104-04$ & $1.488-04$ & $7.329-05$ & $4.212-05$ & $4.571-05$ \\
1 & 76 & $3.341-04$ & $2.524-04$ & $1.965-04$ & $1.181-04$ & $7.927-05$ & $7.663-05$ \\
1 & 77 & $2.922-03$ & $3.098-03$ & $3.220-03$ & $3.419-03$ & $3.556-03$ & $3.293-03$ \\
1 & 78 & $9.913-05$ & $7.592-05$ & $5.991-05$ & $3.669-05$ & $2.445-05$ & $2.239-05$ \\
1 & 79 & $4.460-04$ & $4.480-04$ & $4.662-04$ & $5.432-04$ & $6.342-04$ & $6.100-04$ \\
1 & 80 & $4.826-04$ & $3.716-04$ & $2.937-04$ & $1.804-04$ & $1.204-04$ & $1.088-04$ \\
1 & 81 & $9.907-04$ & $1.268-03$ & $1.550-03$ & $2.213-03$ & $2.818-03$ & $2.684-03$ \\
1 & 82 & $4.913-04$ & $3.815-04$ & $3.050-04$ & $1.870-04$ & $1.246-04$ & $1.104-04$ \\
1 & 83 & $8.312-04$ & $6.499-04$ & $5.256-04$ & $3.348-04$ & $2.351-04$ & $2.144-04$ \\
1 & 84 & $1.149-03$ & $8.878-04$ & $7.092-04$ & $4.339-04$ & $2.888-04$ & $2.558-04$ \\
1 & 85 & $2.827-03$ & $3.371-03$ & $3.842-03$ & $4.774-03$ & $5.459-03$ & $5.185-03$ \\
1 & 86 & $2.117-04$ & $1.487-04$ & $1.082-04$ & $5.655-05$ & $3.389-05$ & $3.243-05$ \\
1 & 87 & $3.078-04$ & $2.337-04$ & $1.869-04$ & $1.310-04$ & $1.089-04$ & $1.054-04$ \\
1 & 88 & $3.779-04$ & $2.664-04$ & $1.937-04$ & $1.011-04$ & $6.047-05$ & $5.813-05$ \\
1 & 89 & $4.967-04$ & $6.000-04$ & $6.949-04$ & $8.724-04$ & $9.883-04$ & $9.474-04$ \\
\hline
\end{tabular}

above. However, for transitions among the excited levels, our estimate of accuracy is $\sim 20 \%$ for a majority of transitions. This estimate is based on a variety of comparisons made among the different calculations.

\section{Effective collision strengths}

Effective collision strengths $\Upsilon$ are obtained after integrating $\Omega$ over a Maxwellian distribution of electron velocities, i.e.

$\Upsilon\left(T_{\mathrm{e}}\right)=\int_{0}^{\infty} \Omega(E) \exp \left(-E_{j} / k T_{\mathrm{e}}\right) \mathrm{d}\left(E_{j} / k T_{\mathrm{e}}\right)$

where $E_{j}$ is the incident energy of the electron with respect to the final state of the transition, $k$ is Boltzmann's constant, and $T_{\mathrm{e}}$ is the electron temperature in $\mathrm{K}$. Once the value of $\Upsilon$ is known for a transition, the corresponding value of the excitation $q(i, j)$ and de-excitation $q(j, i)$ rate coefficients can be easily obtained from the following simple relations:

$q(i, j)=\frac{8.63 \times 10^{-6}}{\omega_{i} T_{\mathrm{e}}^{1 / 2}} \Upsilon \exp \left(-E_{i j} / k T_{\mathrm{e}}\right) \quad \mathrm{cm}^{3} \mathrm{~s}^{-1}$

and

$q(j, i)=\frac{8.63 \times 10^{-6}}{\omega_{j} T_{\mathrm{e}}^{1 / 2}} \Upsilon \quad \mathrm{cm}^{3} \mathrm{~s}^{-1}$,

where $\omega_{i}$ and $\omega_{j}$ are the statistical weights of the initial $(i)$ and final $(j)$ states, respectively, and $E_{i j}$ is the transition energy.

Since the threshold energy region is dominated by numerous resonances, $\Omega$ have been computed at a large number of energies in order to delineate these resonances. We have performed our calculations of $\Omega$ at $\sim 21000$ energies in the threshold region. Close to thresholds ( $\sim 0.1$ Ryd above a threshold) the energy mesh is $0.001 \mathrm{Ryd}$, and away from thresholds is $0.002 \mathrm{Ryd}$.
Thus care has been taken to include as many resonances as possible, and with as fine a resolution as is computationally feasible. The density and importance of resonances can be appreciated from Figs. 6-8 in which we show our $\Omega$ values in the thresholds region for the $1-2\left(2 \mathrm{~s}^{2} 2 \mathrm{p}^{6}{ }^{1} \mathrm{~S}_{0}-2 \mathrm{~s}^{2} 2 \mathrm{p}^{5} 3 \mathrm{~s}^{3} \mathrm{P}_{2}^{\circ}\right), 1-23\left(2 \mathrm{~s}^{2} 2 \mathrm{p}^{6}\right.$ $\left.{ }^{1} \mathrm{~S}_{0}-2 \mathrm{~s}^{2} 2 \mathrm{p}^{5} 3 \mathrm{~d}{ }^{3} \mathrm{D}_{1}^{\circ}\right)$, and $1-27\left(2 \mathrm{~s}^{2} 2 \mathrm{p}^{6}{ }^{1} \mathrm{~S}_{0}-2 \mathrm{~s}^{2} 2 \mathrm{p}^{5} 3 \mathrm{~d}{ }^{1} \mathrm{P}_{1}^{\circ}\right)$ transitions, respectively. Since $1-2(3 \mathrm{H})$ is an important transition, and its resonance structure is very dense, we have shown resonances in Figs. 6a and 6b in both narrow (64-72 Ryd) and wide (64-100 Ryd) energy ranges. It may also be noted that in spite of the 1-23 and 1-27 transitions being allowed, their resonance structures are not insignificant as shown in Figs. 7 and 8. Finally, the resonance structures in Figs. 6-8 are comparable (in magnitude and density) with the similar calculations of Chen et al. (2006a), as shown in their Figs. 1-3.

Our calculated values of $\Upsilon$ are listed in Table 3 over a wide temperature range of $5.2 \leq \log T_{\mathrm{e}} \leq 7.0 \mathrm{~K}$, suitable for applications in astrophysical and other plasmas. As stated in Sect. 1 there is no other similar calculation available in the literature with which to compare our results.

\section{Line ratios}

The intensity of an emission line between the levels $i$ and $j$ is given by

$$
I_{j i}=A_{j i} N_{j} N_{A, Z} N_{A} h v_{j i} \frac{n}{1+N_{\mathrm{He}}} \frac{L}{4 \pi} \quad \quad \operatorname{erg~cm}{ }^{-2} \mathrm{~s}^{-1} \mathrm{sr}^{-1}
$$

where $N_{j}$ is the relative population of level $j, N_{A, Z}$ is the relative ionic abundance of ion with charge $Z$ of element with atomic number $A, N_{A}$ is the relative (with respect to hydrogen) chemical abundance, $N_{\mathrm{He}}$ is the relative chemical abundance of helium, $n$ is the total number density of hydrogen and helium 


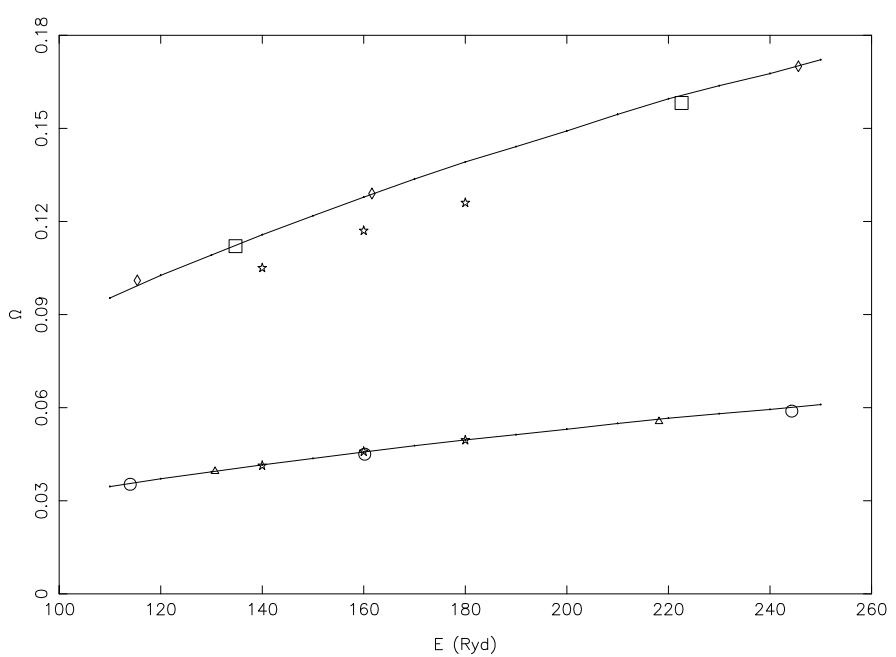

(a)

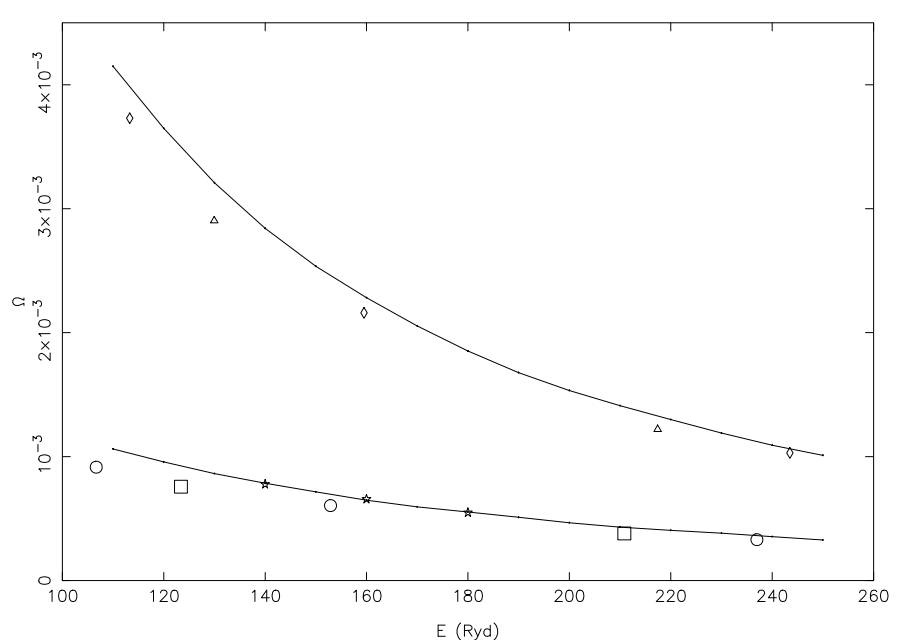

(b)

Fig. 4. a) Comparison of collision strengths from our calculations from DARC for the $1-23\left(2 s^{2} 2 p^{6}{ }^{1} S_{0}-2 s^{2} 2 p^{5} 3 d^{3} D_{1}^{\circ}\right.$ : lower curve $)$ and $1-27$ $\left(2 \mathrm{~s}^{2} 2 \mathrm{p}^{6}{ }^{1} \mathrm{~S}_{0}-2 \mathrm{~s}^{2} 2 \mathrm{p}^{5} 3 \mathrm{~d}^{1} \mathrm{P}_{1}^{\circ}\right.$ : upper curve) transitions of Ni XIX with $\Omega$ from FAC shown as, triangles: $1-23$ and squares: $1-27$ transition. $\Omega$ from DW calculations of Zhang \& Sampson (1989) are shown as, circles: $1-23$ and diamonds: $1-27$ transition. $\Omega$ of Chen et al. (2006a) are shown as stars for both transitions. b) Comparison of collision strengths from our calculations from DARC for the $1-2\left(2 \mathrm{~s}^{2} 2 \mathrm{p}^{6}{ }^{1} \mathrm{~S}_{0}-2 \mathrm{~s}^{2} 2 \mathrm{p}^{5} 3 \mathrm{~s}^{3} \mathrm{P}_{2}^{\circ}\right.$ : lower curve ) and 1-18 (2s $2 \mathrm{~s}^{6}{ }^{1} \mathrm{~S}_{0}-2 \mathrm{~s}^{2} 2 \mathrm{p}^{5} 3 \mathrm{~d}^{3} \mathrm{P}_{2}^{\circ}$ : upper curve $)$ transitions of Ni XIX with $\Omega$ from FAC shown as, squares: $1-2$ and triangles: $1-18$ transition. $\Omega$ from DW calculations of Zhang \& Sampson (1989) are shown as, circles: $1-2$ and diamonds: $1-18$ transition. $\Omega$ of Chen et al. (2006a) are shown as stars for the 1-2 transition.

nuclei (in $\mathrm{cm}^{-3}$ ), and $L$ is the path length through the line emitting region (Kafatos \& Lynch 1980). However, it is difficult to know all of these parameters accurately, and therefore the ratio of two lines eliminates many of these parameters i.e.

$R=\frac{I\left(\lambda_{i j}\right)}{I\left(\lambda_{\mathrm{mn}}\right)}=\frac{A_{j i}}{A_{\mathrm{nm}}} \frac{\lambda_{\mathrm{mn}}}{\lambda_{i j}} \frac{N_{j}}{N_{n}}$

which is a function of atomic parameters alone and does not depend on specific conditions of ionization and recombination. Level populations $N_{j}$ can be calculated in a collisional-radiative model, using for example the statistical equilibrium code of Dufton (1977). The additional advantage of taking the ratio of two lines is that it reduces the error of experimental (observational) data in the interpretation.

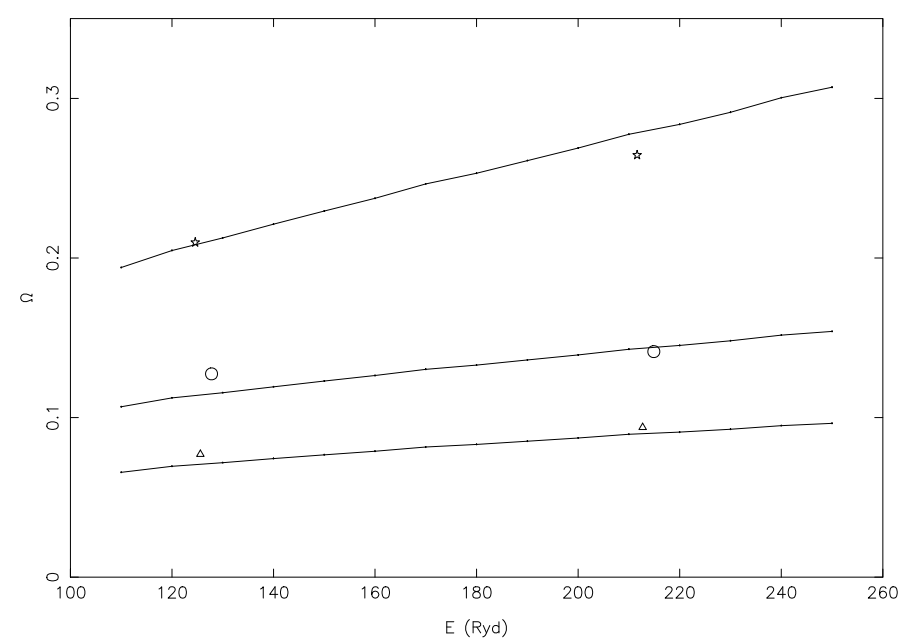

(a)

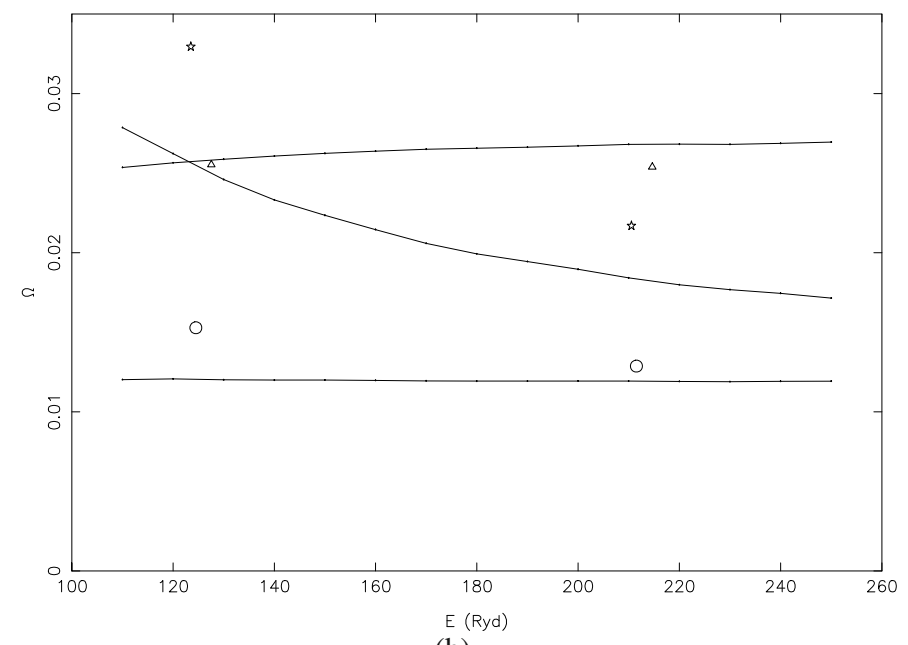

(b)

Fig. 5. a) Comparison of collision strengths from our calculations from DARC (continuous curves) for the $2-13\left(2 \mathrm{~s}^{2} 2 \mathrm{p}^{5} 3 \mathrm{~s}^{3} \mathrm{P}_{2}^{\circ}-2 \mathrm{~s}^{2} 2 \mathrm{p}^{5} 3 \mathrm{p}^{3} \mathrm{P}_{1}\right)$, 5-11 $\left(2 \mathrm{~s}^{2} 2 \mathrm{p}^{5} 3 \mathrm{~s}^{3} \mathrm{P}_{1}^{\circ}-2 \mathrm{~s}^{2} 2 \mathrm{p}^{5} 3 \mathrm{p}^{3} \mathrm{P}_{0}\right)$, and $8-18\left(2 \mathrm{~s}^{2} 2 \mathrm{p}^{5} 3 \mathrm{p}^{3} \mathrm{D}_{3}-2 \mathrm{~s}^{2} 2 \mathrm{p}^{5} 3 \mathrm{~d}\right.$ ${ }^{3} \mathrm{P}_{2}^{\circ}$ ) allowed transitions of Ni XIX with $\Omega$ from FAC shown as, triangles and lower curve: $2-13$, stars and upper curve: $5-11$, and circles and middle curve: 8-18 transition. b) Comparison of collision strengths from our calculations from DARC (continuous curves) for the 2-16 $\left(2 \mathrm{~s}^{2} 2 \mathrm{p}^{5} 3 \mathrm{~s}^{3} \mathrm{P}_{2}^{\circ}-2 \mathrm{~s}^{2} 2 \mathrm{p}^{5} 3 \mathrm{~d}^{3} \mathrm{P}_{0}^{\circ}\right), 6-7\left(2 \mathrm{~s}^{2} 2 \mathrm{p}^{5} 3 \mathrm{p}^{3} \mathrm{~S}_{1}-2 \mathrm{~s}^{2} 2 \mathrm{p}^{5} 3 \mathrm{p}^{3} \mathrm{D}_{2}\right)$, and $10-11\left(2 s^{2} 2 p^{5} 3 p^{3} P_{2}-2 s^{2} 2 p^{5} 3 p^{3} P_{0}\right)$ forbidden transitions of Ni XIX with $\Omega$ from FAC shown as, triangles and upper curve: $2-16$, stars and middle curve: 6-7, and circles and lower curve: 10-11 transition.

The only excitation rates available in the literature are from the calculations of Chen et al. (2006a) for three transitions, namely 1-2 (3H: $\left.2 \mathrm{~s}^{2} 2 \mathrm{p}^{6}{ }^{1} \mathrm{~S}_{0}-2 \mathrm{~s}^{2} 2 \mathrm{p}^{5} 3 \mathrm{~s}{ }^{3} \mathrm{P}_{2}^{\circ}\right), 1-23$ (3D: $2 \mathrm{~s}^{2} 2 \mathrm{p}^{6}$ ${ }^{1} \mathrm{~S}_{0}-2 \mathrm{~s}^{2} 2 \mathrm{p}^{5} 3 \mathrm{~d}^{3} \mathrm{D}_{1}^{\circ}$ ), and 1-27 (3C: $\left.2 \mathrm{~s}^{2} 2 \mathrm{p}^{6}{ }^{1} \mathrm{~S}_{0}-2 \mathrm{~s}^{2} 2 \mathrm{p}^{5} 3 \mathrm{~d}^{1} \mathrm{P}_{1}^{\circ}\right)$. In Table 4 we compare the excitation rates at four common temperatures of $3.0 \times 10^{6}, 5.0 \times 10^{6}, 1.0 \times 10^{7}$, and $1.2 \times$ $10^{7} \mathrm{~K}$. Also included in this table are the excitation rates for three other transitions, namely 1-3 (3G: $2 \mathrm{~s}^{2} 2 \mathrm{p}^{6}{ }^{1} \mathrm{~S}_{0}-2 \mathrm{~s}^{2} 2 \mathrm{p}^{5} 3 \mathrm{~s}$ ${ }^{1} \mathrm{P}_{1}^{\circ}$ ), 1-5 (3F: $\left.2 \mathrm{~s}^{2} 2 \mathrm{p}^{6}{ }^{1} \mathrm{~S}_{0}-2 \mathrm{~s}^{2} 2 \mathrm{p}^{5} 3 \mathrm{~s}^{3} \mathrm{P}_{1}^{\circ}\right)$, and 1-17 (3E: $2 \mathrm{~s}^{2} 2 \mathrm{p}^{6}$ $\left.{ }^{1} \mathrm{~S}_{0}-2 \mathrm{~s}^{2} 2 \mathrm{p}^{5} 3 \mathrm{~d}^{3} \mathrm{P}_{1}^{\circ}\right)$. Additionally, the ratios $3 \mathrm{C} / 3 \mathrm{D}, 3 \mathrm{C} / 3 \mathrm{E}$, and $3 \mathrm{C} /(3 \mathrm{~F}+3 \mathrm{G}+3 \mathrm{H})$ are also listed for a ready comparison with other theoretical data. Corresponding experimental results for these line ratios have been shown by Gu et al. (2004) in their Fig. 3. For the convenience of readers, and to facilitate a ready comparison, we list their ratios in Table 5, from both spectrometer and calorimeter measurements. The uncertainties in their 


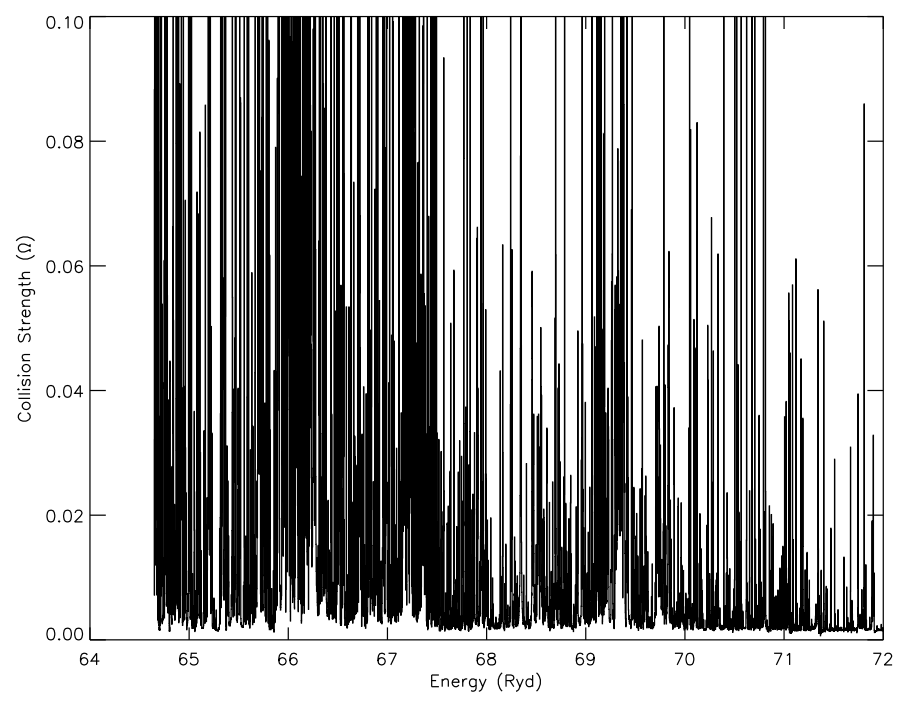

(a)

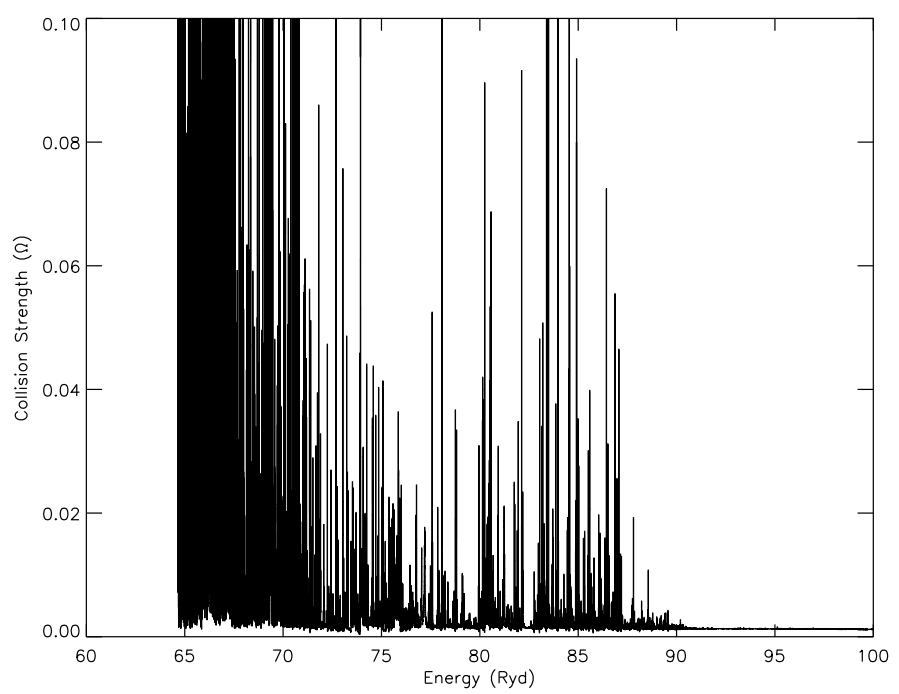

(b)

Fig. 6. Collision strengths for the $2 s^{2} 2 p^{6}{ }^{1} \mathrm{~S}_{0}-2 s^{2} 2 p^{5} 3 s^{3} \mathrm{P}_{2}^{\circ}$ (3H: 1-2) transition of Ni XIX and for the $2 \mathrm{~s}^{2} 2 \mathrm{p}^{6}{ }^{1} \mathrm{~S}_{0}-2 \mathrm{~s}^{2} 2 \mathrm{p}^{5} 3 \mathrm{~s}^{3} \mathrm{P}_{2}^{\circ}(3 \mathrm{H}$ : 1-2) transition of Ni XIX.

measurements are $9 \%, 19 \%$, and $16 \%$ for $3 \mathrm{C} / 3 \mathrm{D}, 3 \mathrm{C} / 3 \mathrm{E}$, and $3 \mathrm{C} /(3 \mathrm{~F}+3 \mathrm{G}+3 \mathrm{H})$, respectively. However, the uncertainty for the $3 \mathrm{C} / 3 \mathrm{E}$ line ratio is $23 \%$ for measurements from the calorimeter.

Our $3 \mathrm{C} / 3 \mathrm{D}$ ratio varies by $10 \%$ over the entire temperature range, similar to the variation in the data of Chen et al. (2006a). This is because both the 3C and 3D lines are allowed, and the effect of resonances on excitation rates is not prominent as seen in Figs. 7 and 8. However, our 3C/3D ratios are higher by $\sim 10 \%$ than those of Chen et al. This is mainly because their values of $\Omega$ (and subsequently of excitation rates) are lower for the 3C transition as seen earlier in Fig. 4a. The corresponding ratios from the $\Omega$ values of Zhang \& Sampson (1989) and FAC are $\sim 2.85$, which are up to $17 \%$ higher than our results from DARC and up to $28 \%$ higher than those of Chen et al. A more accurate value of $3 \mathrm{C} / 3 \mathrm{D}$ from FAC is $\sim 2.56$ as determined by $\mathrm{Gu}$ et al. (2004), who have included the effect of resonances through the isolated resonance approximation as well as of cascading from higher excited levels. This ratio by $\mathrm{Gu}$ et al. is closer to our own calculations than those of

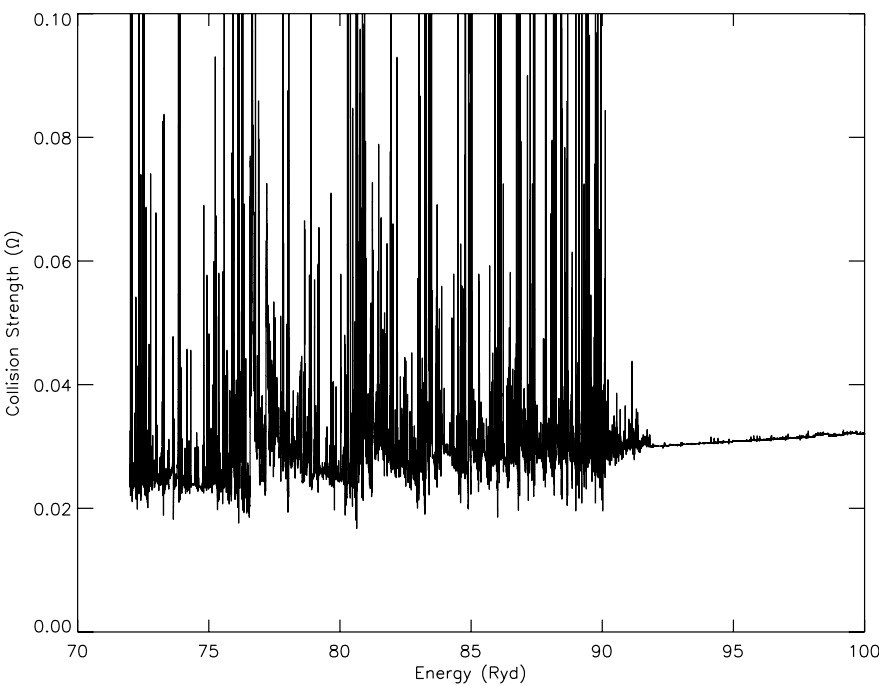

Fig. 7. Collision strengths for the $2 s^{2} 2 p^{6}{ }^{1} S_{0}-2 s^{2} 2 p^{5} 3 d^{3} D_{1}^{\circ}$ (3D: $1-23$ ) transition of Ni XIX.

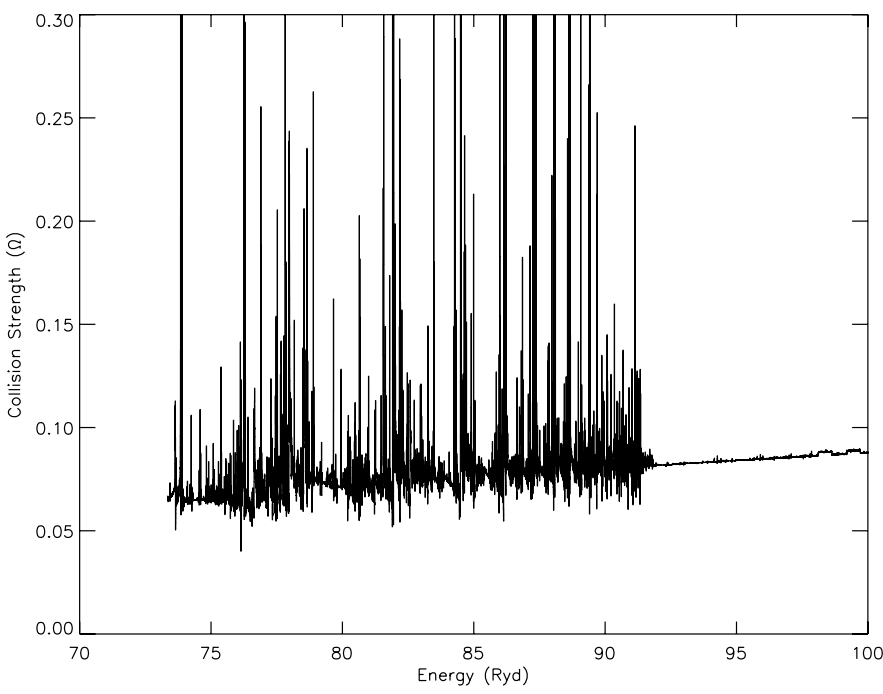

Fig. 8. Collision strengths for the $2 s^{2} 2 p^{6}{ }^{1} S_{0}-2 s^{2} 2 p^{5} 3 d{ }^{1} P_{1}^{\circ}(3 C: 1-27)$ transition of Ni XIX.

Chen et al., and the discrepancy with the experimental value of $2.30 \pm 0.16$ (Brown et al. 2001) remains up to $10 \%$, depending on the temperature. The experimental ratio of Brown et al. has been reconfirmed by $\mathrm{Gu}$ et al. through measurements with a grating spectrometer. However, their similar measurements from an Xray calorimeter give $3 C / 3 \mathrm{D}$ ratio lower by $\sim 10 \%$, but Chen et al. (2006b) measure this to be $2.29 \pm 0.16$ from a microcalorimeter on the EBIT machine. Therefore, considering the upper limits of measurements, i.e. 2.30 with an uncertainty of $9 \%$, we may also state that there is no discrepancy between theory and experiment for the ratio of $3 \mathrm{C}$ and $3 \mathrm{D}$ lines. However, the corresponding theoretical results of Chen et al. (2006a), although in agreement with the measurements, are apparently underestimated, and their assessment of $5 \%$ accuracy does not appear to be realistic. We discuss this further below.

The theoretical ratios discussed above are based on the assumption that the electron velocity distribution in a plasma is Maxwellian. However, the EBIT measurements are carried out with a "monoenergetic" electron beam with an electron distribution function assumed to be "Gaussian", with characteristic beam width between 10 and $30 \mathrm{eV}$ 
Table 4. Comparison of excitation rates and line intensity ratios for some transitions of Ni XIX. $\left(a \pm b \equiv a \times 10^{ \pm b}\right)$.

\begin{tabular}{|c|c|c|c|c|c|c|c|c|c|}
\hline \multirow{2}{*}{$\begin{array}{l}\text { Line } \\
\text { Label }\end{array}$} & \multirow{2}{*}{$\begin{array}{l}\text { Transition } \\
\text { Temperature }\end{array}$} & \multicolumn{4}{|c|}{ Present results } & \multicolumn{4}{|c|}{ "Chen et al. (2006a) } \\
\hline & & $3.0 \times 10^{6}$ & $5.0 \times 10^{6}$ & $1.0 \times 10^{7}$ & $1.2 \times 10^{7}$ & $3.0 \times 10^{6}$ & $5.0 \times 10^{6}$ & $1.0 \times 10^{7}$ & $1.2 \times 10^{7} \mathrm{~K}$ \\
\hline $3 \mathrm{H}$ & $1-2$ & $1.74-12$ & $3.59-12$ & $4.10-12$ & $3.84-12$ & $1.69-12$ & $3.47-12$ & $4.03-12$ & $3.79-12$ \\
\hline $3 \mathrm{G}$ & $1-3$ & $2.40-12$ & $5.36-12$ & $7.51-12$ & $7.52-12$ & & & & \\
\hline $3 \mathrm{~F}$ & $1-5$ & $2.06-12$ & $4.53-12$ & $6.14-12$ & $6.10-12$ & & & & \\
\hline $3 \mathrm{E}$ & $1-17$ & $7.11-13$ & $2.11-12$ & $3.53-12$ & $3.59-12$ & & & & \\
\hline $3 \mathrm{D}$ & $1-23$ & $3.69-12$ & $1.38-11$ & $3.35-11$ & $3.72-11$ & $3.53-12$ & $1.34-11$ & $3.46-11$ & $4.02-11$ \\
\hline $3 \mathrm{C}$ & $1-27$ & $8.98-12$ & $3.55-11$ & $8.97-11$ & $1.00-10$ & $7.88-12$ & $3.10-11$ & $8.29-11$ & $9.76-11$ \\
\hline \multicolumn{2}{|c|}{$3 \mathrm{C} / 3 \mathrm{D}$} & $2.43^{a}$ & $2.57^{a}$ & $2.68^{a}$ & $2.69^{a}$ & $2.23^{a}$ & $2.31^{a}$ & $2.40^{a}$ & $2.43^{a}$ \\
\hline \multicolumn{2}{|c|}{$3 \mathrm{C} / 3 \mathrm{D}$} & $2.38^{b}$ & $2.50^{b}$ & $2.60^{b}$ & $2.61^{b}$ & & & & \\
\hline \multicolumn{2}{|c|}{$3 \mathrm{C} / 3 \mathrm{E}$} & $12.63^{a}$ & $16.82^{a}$ & $25.41^{a}$ & $27.86^{a}$ & & & & \\
\hline \multicolumn{2}{|c|}{$3 \mathrm{C} / 3 \mathrm{E}$} & $13.10^{b}$ & $16.90^{b}$ & $24.40^{b}$ & $27.80^{b}$ & & & & \\
\hline \multicolumn{2}{|c|}{$3 \mathrm{C} /(3 \mathrm{~F}+3 \mathrm{G}+3 \mathrm{H})$} & $0.31^{b}$ & $0.42^{b}$ & $0.60^{b}$ & $0.64^{b}$ & & & & \\
\hline
\end{tabular}

${ }^{a}$ Ratios from excitation rates; ${ }^{b}$ ratios from collisional radiative (CR) model.

Table 5. Experimentally measured line intensity ratios for some transitions of Ni XIX (Gu et al. 2004).

\begin{tabular}{lllrrrl}
\hline \hline Energy & \multicolumn{2}{c}{$3 \mathrm{C} / 3 \mathrm{D}$} & \multicolumn{2}{c}{$3 \mathrm{C} / 3 \mathrm{E}$} & \multicolumn{2}{c}{$3 \mathrm{C} /(3 \mathrm{~F}+3 \mathrm{G}+3 \mathrm{H})$} \\
$\mathrm{KeV}$ & Spectrometer & Calorimeter & Spectrometer & Calorimeter & Spectrometer & Calorimeter \\
\hline 1.043 & 2.354 & 2.074 & 9.152 & 7.980 & 0.401 & 0.422 \\
1.137 & 2.236 & 1.921 & 9.258 & 7.129 & 0.418 & 0.434 \\
1.238 & 2.251 & 2.198 & 11.919 & 9.814 & 0.407 & 0.456 \\
1.328 & 2.303 & 2.177 & 12.963 & 12.033 & 0.448 & 0.467 \\
1.442 & 2.350 & 2.110 & 18.972 & 12.036 & 0.461 & 0.486 \\
\hline
\end{tabular}

(Chen et al. 2006a,b). Brown et al. (2001), on the other hand, have specified that their measurements are equivalent to both Maxwellian and non-Maxwellian plasmas. Nevertheless, calculations performed with a Gaussian distribution yield comparable ratios to the Maxwellian distribution, as demonstrated by Chen et al. Therefore, the choice of electron energy distribution does not affect the line ratios and/or the discrepancy between theory and measurements.

The $3 \mathrm{C} / 3 \mathrm{E}$ line ratio has a larger variation of over a factor of two over the temperature range $3.0 \times 10^{6} \leq T_{\mathrm{e}} \leq 1.2 \times 10^{7} \mathrm{~K}$, as shown in Table 4. Chen et al. (2006a, 2006b) have not reported their results for this ratio, but the corresponding ratio from the $\Omega$ values of Zhang \& Sampson (1989) varies between 31 and 57, and from our calculations from FAC between 42 and 98. Therefore, the variation factor from the lowest to highest ratio remains around the same in all calculations. We would also like to note here that the $1-17$ (3E) transition is an intercombination one, its resonance structure is not very prominent, and therefore the contribution of resonances in the determination of excitation rates is insignificant over the temperature range discussed here. The experimental ratios vary between 9.15 and 18.97 in the energy range $1.04 \leq E \leq 1.44 \mathrm{KeV}$ from the spectroscopic measurements, and between 7.98 and 12.04 from the calorimeter measurements ( $\mathrm{Gu}$ et al. 2004), and have a higher uncertainty of $19 \%$. Therefore, at higher energies (temperatures) there appears to be an agreement between the spectroscopic measurements and our calculations from DARC, but the similar measurements from calorimeter are underestimated by up to $\sim 40 \%$, particularly at the highest energy, i.e. $1.44 \mathrm{KeV}$. As for the line ratio $3 \mathrm{C} / 3 \mathrm{D}$, a more accurate determination of $3 \mathrm{C} / 3 \mathrm{E}$, by including the effects of resonances and cascading, is $\sim 20$ ( $\mathrm{Gu}$ et al.). This value of $3 \mathrm{C} / 3 \mathrm{E}$ is overestimated by up to $70 \%$ in comparison to measurements and is underestimated by up to $40 \%$ in comparison to our calculations from DARC.
As for $3 \mathrm{C} / 3 \mathrm{E}$, the theoretical $3 \mathrm{C} /(3 \mathrm{~F}+3 \mathrm{G}+3 \mathrm{H})$ ratio also varies by a factor of two over the temperature range $3.0 \times 10^{6} \leq$ $T_{\mathrm{e}} \leq 1.2 \times 10^{7} \mathrm{~K}$, as shown in Table 4. However, the experimental ratio only varies by $\sim 15 \%$ between 1.0 and $1.44 \mathrm{KeV}$ as can be noted from Table 5, i.e. it may be considered to be energy independent if we take into consideration the uncertainty of measurements. Over the same energy range, the nonresonant calculations of $\mathrm{Gu}$ et al. (2004) show a variation of less than $30 \%$, but the ratio with resonances included varies up to $50 \%$, and the lowest value is at the lowest energy. An average value of $3 \mathrm{C} /(3 \mathrm{~F}+3 \mathrm{G}+3 \mathrm{H})$ from the calculations of $\mathrm{Gu}$ et al., which includes the contribution of resonances and cascading, may be considered to be $\sim 0.55$. This ratio is upto $80 \%$ higher at lower temperatures and up to $15 \%$ lower at higher temperatures than our corresponding results from DARC. Both the spectrometer and calorimeter give a consistent value $(\sim 0.46)$ for this ratio, which is up to $30 \%$ lower (at higher energies/temperatures) than the corresponding theoretical results. The lower values of the $3 \mathrm{C} /(3 \mathrm{~F}+3 \mathrm{G}+3 \mathrm{H})$ ratio at lower temperatures, particularly from our calculations from DARC, are due to the fact that all the three lines (i.e. $3 \mathrm{~F}, 3 \mathrm{G}$, and $3 \mathrm{H}$ ) show a dominant contribution from resonances, as can be noted from Fig. 6 for the $1-2(3 \mathrm{H})$ transition.

\section{Conclusions}

In the present work, we have reported results for collision strengths and effective collision strengths for transitions among the 89 levels of Ni XIX over a wide energy (temperature) range up to 250 Ryd $\left(10^{7} \mathrm{~K}\right)$. The relativistic DARC code has been adopted for the calculations, and a large range of partial waves has been included in order to achieve converged values of collision strengths. Furthermore, resonances have been resolved in a fine energy mesh in thresholds region in order to account for 
their contribution in the determination of $\Upsilon$ values. The presently reported results for $\Upsilon$ are the only data available to date for all transitions among the 89 levels of Ni XIX. These results, together with our earlier reported (Aggarwal \& Keenan 2006b) radiative rates for four types of transitions, namely E1, E2, M1, and M2, should be highly useful for the modelling of a variety of plasmas.

Based on a variety of comparisons, among different calculations, the accuracy of our $\Omega$ and $\Upsilon$ values is assessed to be better than $10 \%$ for all resonance transitions. However, the accuracy assessment for other transitions, among the excited levels, is $\sim 20 \%$. Similarly, there is a general agreement among various calculations for the $3 \mathrm{C} / 3 \mathrm{D}$ line intensity ratio, and there is no discrepancy with the experiments either. However, differences between theory and experiment for the $3 \mathrm{C} / 3 \mathrm{E}$ and $3 \mathrm{C} /(3 \mathrm{~F}+3 \mathrm{G}+3 \mathrm{H})$ ratios remain, for which further work may be helpful. Additionally, the energy (temperature) variations between theory and experiments for the $3 \mathrm{C} / 3 \mathrm{E}$ line ratios are comparable. However, for the $3 \mathrm{C} /(3 \mathrm{~F}+3 \mathrm{G}+3 \mathrm{H})$ ratios experimental values are (almost) energy independent, but the theoretical ones show large variations with temperature.

Acknowledgements. This work has been financed by the Engineering and Physical Sciences Research Council (EPSRC) and the Science and Technology Facilities Council (STFC) of the United Kingdom. We would like to thank Dr. Patrick Norrington for providing his GRASP and DARC codes prior to publication, and to Dr. Ming Gu for providing his experimental and theoretical line ratios in numerical form. Finally, F.P.K. is grateful to AWE Aldermaston for the award of a William Penney Fellowship.

\section{References}

Aggarwal, K. M., \& Keenan, F. P. 2006a, Pramana - J. Phys., 67, C553 Aggarwal, K. M., \& Keenan, F. P. 2006b, A\&A, 460, 959

Aggarwal, K. M., \& Keenan, F. P. 2007a, Pramana - J. Phys., 69, 209

Aggarwal, K. M., \& Keenan, F. P. 2007b, J. Phys. Conf. Ser., 58, 169

Aggarwal, K. M., \& Keenan, F. P. 2007c, A\&A, 475, 393
Aggarwal, K. M., \& Keenan, F. P. 2008, Eur. Phys. J. D, 46, 205

Aggarwal, K. M., Keenan, F. P., \& Msezane, A. Z. 2003, At. Data Nucl. Data Tables, 85, 453

Aggarwal, K. M., Tayal, V., Gupta, G. P., \& Keenan, F. P. 2007, At. Data Nucl. Data Tables, 93, 615

Aggarwal, K. M., Hamada, K., Igarashi, A., et al. 2008, A\&A, 484, 879

Berrington, K. A., Burke, P. G., Le Dourneuf, M., et al. 1978 Comput. Phys. Commun., 14, 367

Biémont, E., Magunov, A. I., Dyakin, V. M., et al. 2000, J. Phys. B, 33, 2153

Boiko, V. A., Pikuz, S. A., Safronova, U. I., \& Faenov, A. Y. 1977, J. Phys. B, 10,1253

Brown, G. V., Beiersdorfer, P., \& Widmann, K. 2001, Phys. Rev. A 63, 032719

Buchet, J. P., Buchet-Poulizac, M. C., Denis, A., et al. 1987, J. Phys. B, 20, 1709

Burgess, A., Hummer, D. G., \& Tully, J. A. 1970, Phil. Trans. Roy. Soc., 266 A, 225

Chen, G. X., Kirby, K., \& Brickhouse, N. S. 2006a, Phys. Rev. A, 73, 052708

Chen, G. X., Kirby, K., Silver, E., et al. 2006b, Phys. Rev. Lett., 97, 143201

Del Zanna, G., Berrington, K. A., \& Mason, H. E. 2004, A\&A, 422, 731

Dufton, P. L. 1977, Comput. Phys. Commun., 13, 25

Feldman, U., Cohan, L., \& Swartz, M. 1967, ApJ, 148, 585

Feldman, U., Curdt, W., Landi, E., \& Wilhelm, K. 2000, ApJ, 544, 508

Grant, I. P., McKenzie, B. J., Norrington, P. H., Mayers, D. F., \& Pyper, N. C. 1980, Comput. Phys. Commun., 21, 207

Gu, M. F. 2003, ApJ, 582, 1241

Gu, M. F., Beiersdorfer, P., Brown, G. V., et al. 2004, ApJ, 607, L143

Gu, M. F., Beiersdorfer, P., Brown, G. V., et al. 2007, ApJ, 657, 1172

Hibbert, A., Le Dourneuf, M., \& Mohan, M. 1993, At. Data Nucl. Data Tables, 53,23

Jupen, C. 1984, MNRAS, 208, 1P

Kafatos, M., \& Lynch, J. P. 1980, ApJS, 42, 611

Krongold, Y., Nicastro, F., Brickhouse, N. S., et al. 2003, ApJ, 597, 832

Lucy, L. B. 1995, A\&A, 294, 555

Miller, J. M., Wojdowski, P., Schulz, N. S., et al. 2005, ApJ, 620, 398

Mohan, M., Le Dourneuf, M., Hibbert, A., \& Burke, V. M. 1993, Pramana - J. Phys., 41, 371

Reeves, J. N., Watson, D., Osborne, J. P., et al. 2002, Nature, 416, 512

Singh, N., \& Mohan, M. 2005, Pramana - J. Phys., 64, 129

Swartz, M., Kastner, S., Rothe, E., \& Neupert, W. 1971, J. Phys. B, 4, 1747

Zhang, H. L., \& Sampson, D. H. 1989, At. Data Nucl. Data Tables, 43, 1

Zhang, H. L., Sampson, D. H., Clark, R. E. H., \& Mann, J. B. 1987, At. Data Nucl. Data Tables, 37, 17 